\title{
Multi-Segment Deep Convolution Neural Networks for Classification of Faults in Sensors at Railway Point Systems \\ DOI:
}

10.23919/IConAC.2019.8895081

\section{Document Version}

Accepted author manuscript

Link to publication record in Manchester Research Explorer

Citation for published version (APA):

Sachan, S., \& Donchak, N. (2019). Multi-Segment Deep Convolution Neural Networks for Classification of Faults in Sensors at Railway Point Systems. In 25th IEEE International Conference on Automation and Computing (pp. 1). IEEE. https://doi.org/10.23919/IConAC.2019.8895081

\section{Published in:}

25th IEEE International Conference on Automation and Computing

\section{Citing this paper}

Please note that where the full-text provided on Manchester Research Explorer is the Author Accepted Manuscript or Proof version this may differ from the final Published version. If citing, it is advised that you check and use the publisher's definitive version.

\section{General rights}

Copyright and moral rights for the publications made accessible in the Research Explorer are retained by the authors and/or other copyright owners and it is a condition of accessing publications that users recognise and abide by the legal requirements associated with these rights.

\section{Takedown policy}

If you believe that this document breaches copyright please refer to the University of Manchester's Takedown Procedures [http://man.ac.uk/04Y6Bo] or contact uml.scholarlycommunications@manchester.ac.uk providing relevant details, so we can investigate your claim.

\section{OPEN ACCESS}




\section{Multi-Segment Deep Convolution Neural Networks for Classification of Faults in Sensors at Railway Point Systems}

\author{
Swati Sachan \\ Decision and Cognitive Research Centre \\ Alliance Manchester Business School \\ The University of Manchester \\ Booth Street West \\ Manchester, M15 6PB \\ swati.sachan@manchester.ac.uk
}

\author{
Nishant Donchak \\ Senior Managing Consultant \\ Berkeley Research Group \\ 8 Salisbury Square \\ London \\ EC4Y 8AP \\ ndonchak@,thinkbrg.com
}

\begin{abstract}
Intelligent fault detection by sensor data can ensure the reliability and availability of critical infrastructures. Switches and crossings (S\&C) are one of the most important assets of railway networks. They divert trains in different directions by shifting the position of switch rail by point operating equipment (POE). The sensors record the electrical current drawn by the motor in POE. The extraction of features from time-series sensor data enables the detection of faults in POE. This paper proposes a deep learning model to detect faults in railway POE without the need for preprocessing the raw time-series data. It is based on 1-D convolution neural network. The architecture of the proposed deep learning network consists of three types of layers. The first layer is called the local convolution layer. It consists of three 1-D convolution layer to extract local temporal features from three non-overlapping segments of time-series data of different operating phases of POE. The second layer is fully-connected convolution layer. It extracts global temporal features. And the last layer is the output layer, it provides the binary output of fault or fault free for a given sensor data. The result shows that this framework can classify fault with $95.60 \%$ accuracy.
\end{abstract}

Keywords - deep learning; convolution neural network; fault; classfication; railway

\section{INTRODUCTION}

\section{A. Railway Switches \& Crossing}

Asset holder implements various maintenance policy to ensure reliability and availability of critical infrastructure to provide continuous services. Railways are large scale and spatially distributed infrastructures. Switches and crossings (S\&C) are one of the most important assets of railway networks. $\mathrm{S} \& \mathrm{C}$ are the devices that allow trains to cross tracks and continue in a different direction or in the same direction in different tracks [1]. They are crucial in diverting the train to other track when it is blocked by traffic congestion or line failures. The layout of railway $\mathrm{S} \& \mathrm{C}$ is shown in Figure 1. The S\&C consist of four types of rail stock rail, switch rail, wing rail and check rail. Stock rail is fixed rail, it ensures the continuity of the straight main or diverted track depending upon an open position of the switch. Switch rail is movable rail. The S\&C driving devices push the switch rail accurately to the desired position to allow passage of trains. S\&C has moving parts and failures of these parts lead to train delays, cancellations and sometimes derailments. The failures of S\&C have a direct effect on the quality of service and has large financial implications.

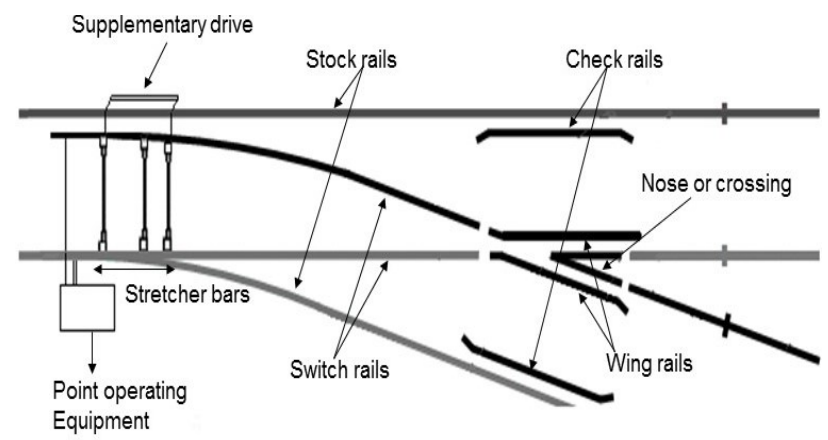

Figure 1. Layout of railway switches and crossing

\section{B. Point Operating Equipment}

The purpose of point operating equipment (POE) is to push the switch rail towards the stock rail without the gap. There are many types of POE. In this study POE with clamp locks are considered. A POE unit consists of two cast locks (left-hand and right-hand side) and detectors, two hydraulic actuators and a flexible hose connected to a separately mounted electro-hydraulic motor. The movement of the points is achieved by two hydraulic actuators connected to the centrally mounted thrusters and drive lock slides. The relay put the feed into the power pack as shown in Figure 2. POE has four operating phases, shown in Figure 3:

- Motor initialization: Pump motor starts when signal is received to change the direction by railway point system.

- Unlocking mechanism: Switch rail are unlocked

- Switch rail movement: Pump motor pumps the hydraulic fluid into retracting actuator. The retracting 
actuator pushes the drive lock slides to move the switch rails.

- Locking mechanism: After completion of switch rail movement, the switch rails are locked at the new position and the signal is send to railway point system that it has successfully complete the operation. The motor keeps running due to slight delay in recognition of locking of switch rail.

The clamp lock has the highest number of failures and their failure is one of the main cause of train delays [2]. The faults and defects in the clamp lock system are detected by utilizing condition monitoring techniques and practicing detailed inspection, respectively. Currently, most point equipment condition monitoring system has only one type of sensors which can record the electrical current drawn by the motor [2][3]. The alarm in the condition monitoring system triggers when the observed parameter exceeds the threshold value. The threshold value is predetermined and chosen based on expert knowledge. The data collected from data logging sensors are transmitted to the central data server, where electrical current trend variation is studied and compared by statistical or machine learning methods to detect the fault in the clamp lock operation. In past statistical methods [3][4][5][6], classification by machine learning [7][8] and expert systems [9] has been presented to detect fault in railway point operating systems. The classification by machine learning methods has shown promising results.

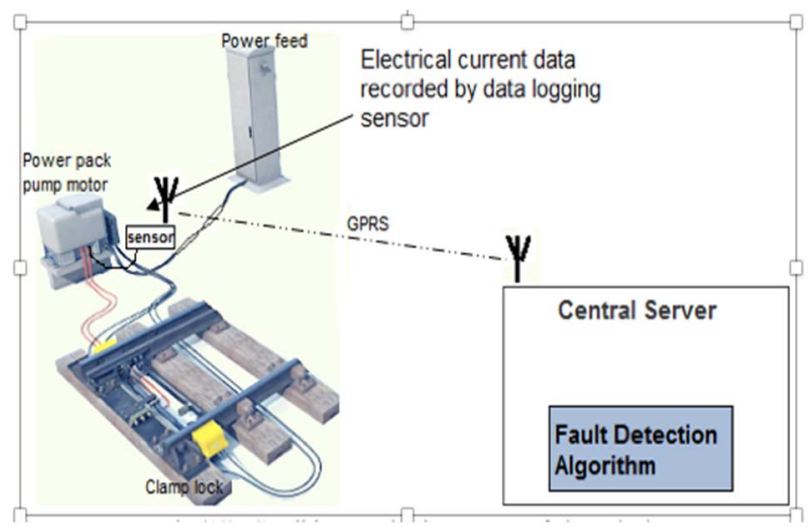

Figure 2. Condition monitoring system of POE and sensor data collection

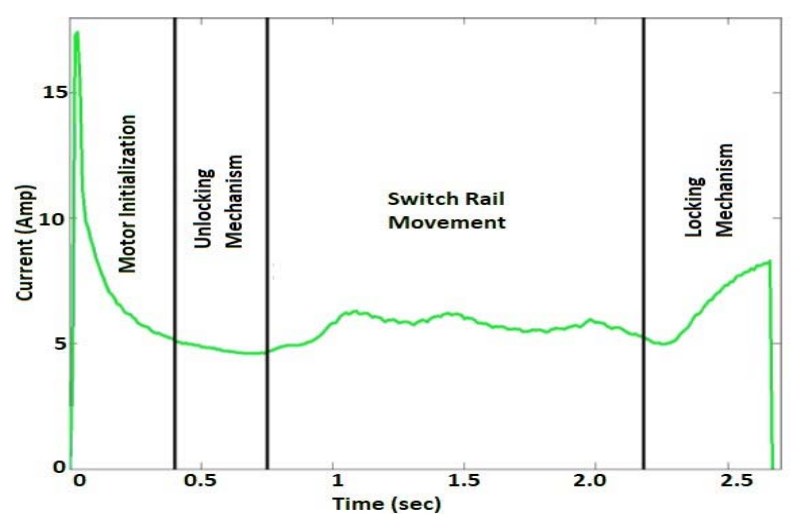

Figure 3. Operating phases of point operating equipment

\section{Related Work-Classification of Time Series by Deep Learning}

Fault detection in POE by one class support vector machine (SVM) was presented in [2]. This method requires time series data processing by uniform scaling, exponential smoothing, and downscaling which necessitates adequate knowledge of signal processing.

Recently, efforts have been put to exploit deep learning methods to classify time series data [10]. Convolution neural network (CNN) is especially deployed for end-toend time series classification. In [11], multi-channel CNN (MCCNN) is proposed for classification of multivariate time series data. It applies independent convolution on each channel. In the later phase, all independent convolutions are concatenated which is followed by a fully connected convolution layer. In another paper [12], authors proposed multi-scale CNN (MCNN) for classification of univariate time-series data. This framework has local convolution stage in which multiple down-sample, low frequency and original time series are independently convoluted. In the next full convolution stage, all independent convolutions are concatenated for full convolution. This method requires heavy data processing efforts. In [13] and [14] authors presented an integrated convolution and recurrent neural network architecture to capture the temporal relationships from raw time series data for mobile sensing and wearable devices, respectively. A comprehensive comparison of benchmark methods with new frameworks for classification of time series data by deep neural networks is presented in [10]. It reports that the fully convolutional networks (FCN) achieve good performance without heavy processing of raw data. The residual neural network (ResNet) give competitive performance compared to other frameworks. However, the main focus of ResNet is on visual inputs, they may not lose the capacity to capture temporal relationship which is important for time-series data from sensors.

The rest of paper is organized as follows. Section II, describes the data collection and data segmentation of sensor data. Section III, describes the architecture of multisegmentation deep convolution neural network which includes three types of layers - local convolution and pooling layers, full convolution and pooling layers, and output layer. At the end of this section discuss the methodology for hyperparameter optimization and training of this deep learning model. The results are shown in Section IV. The paper is concluded in Section V.

\section{DATA COLLECTION AND DATA SEGMENTATION}

The data was collected from the sensor in HW1000/2000 series, a type of point operating equipment at the UK railway network. The sensor at POE measures a single time-varying electrical current signal. It samples data at $100 \mathrm{~Hz}$ (sensor delay of 10,000 microseconds). The dataset contains data of 1361 movements of the year 2016. Out of 1361 data samples, there were 200 faulty movements and the rest of the movements were fault free.

The input in the deep-learning model is raw timedomain data instead of processed data in time domain or 
frequency domain. The proposed deep convolution neural network is structured to learn local and global temporal features from raw time-varying electrical current data. The length of time-series is denoted by $T$ (sec). A timevarying current data is denoted by matrix $K$, where $K=$ $\tau \times \beta, \tau$ denotes the number of measurements (timestamps) within time period $T$ and $\beta$ denotes the number of sensors. In this case, POE has only one sensor, $\beta=1$. We split the number of measurements into $l$ segments to obtain a series of non-overlapping time-interval, as shown in Figure 4 . The size of each segment is equivalent to the number of operating phases of POE. In this study, motor initiation phase is ignored. The electrical current from time $0.5 \mathrm{sec}$ to $3 \mathrm{sec}$ is considered, therefore, the length of time series is $T=3-0.5=2.5 \mathrm{sec}$. The size of the segment and the number of data points in each segment is shown in Table 1.

\begin{tabular}{|c|c|c|}
\hline \multirow{2}{*}{ Segment } & \multicolumn{2}{|c|}{ Table 1: Data Segmentation } \\
\cline { 2 - 3 } & Time series segment & Number of measurements \\
\hline 1 & 0.50 to $0.75 \mathrm{sec}$ & 23 \\
\hline 2 & 0.76 to $2.20 \mathrm{sec}$ & 140 \\
\hline 3 & 2.21 to $3.00 \mathrm{sec}$ & 80 \\
\hline
\end{tabular}

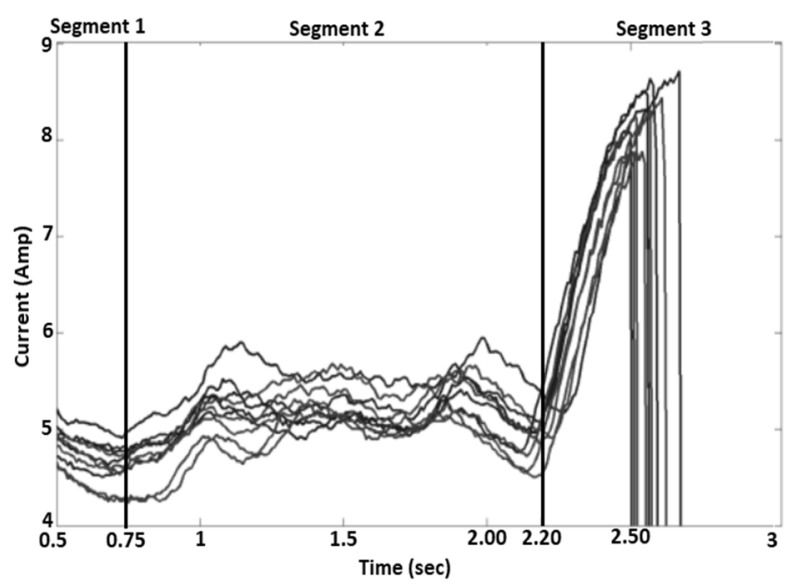

Figure 4. Data Segmentation

\section{Architecture of Multi- Segment CONVOLUTION NEURAL NETWORKS}

The proposed deep learning framework for fault detection in railway point system has three types of layers; the local convolution and pool layers, the full convolution and pooling layers, and the output layer. The architecture of the multi-segment convolutional neural network is shown in Figure 5. It utilizes 1-D CNN in both local and full-convolution and pooling layers. 1-D CNN is very effective in extracting temporal dependencies from short and fixed-length time-series data [13]. The filters in 1-D CNN slide only in one-direction either vertically from topto-bottom or horizontally from left-to-right. 2-D CNN is effective for images, where filter slides in both horizontal and vertical direction. These layers are discussed in the following subsection.

\section{A. Local Convolution and pooling layers}

Independent 1-D convolution is applied to each segment of time-series data. Three independent convolution layers extract local features from each segment of different operating phases of POE. The size of the input (height $\times$ width) for independent convolution for segment 1 , segment 2 , and segment 3 is $23 \times 1,140 \times$ 1 , and $80 \times 1$, respectively. The filter (feature extractor) of size $f=3$ is same across all local convolution layers and each layer learns from 50 filters $(\# f=50)$ and uses rectified linear unit ( $\mathrm{ReLu})$ activation function. The stride that is steps of scanning the features vertically in each local convolution is equal to $s=1$. All three local convolution layers are followed by a maximum pooling layer. Maximum pool selects the largest element within the region covered by the filter. The pooling layer does not have any parameter to learn. It is often used after convolution layer to reduce complexity and prevent overfitting of learned features. For time-series data, it is a form of non-linear down-sampling. The filter of size $f=$ 2 and stride $s=2$ is used for maximum pooling of output from local convolution layer.

\section{B. Full Convolution and pooling layers}

The output from the local convolution and pooling layer is concatenated to use it as the input in the next full convolution layer. All features are concatenated vertically. The size of the concatenated matrix of heterogeneous set convolution and pooled layer is $116 \times 50$. This concatenated layer is scanned by 25 filters of size $f=3$ with stride $s=1$. It gives an output matrix of size $112 \times$ 25 . The full convolution is followed by maximum pooling layer with $\mathrm{f}=2$ and $s=2$, which give an output of size $56 \times 25$.

\section{Output layer}

The output from the previous maximum pooling of full convolution $(56 \times 25)$ is flattened to make two fully connected or dense layers of size 1400 . Both dense layers are followed by a dropout connection to reduces the overfitting. A fraction of neurons is randomly set to zero. The dropout rate in this deep learning model is 0.30 , that is, $30 \%$ of neurons will be dropped randomly. The final fully connected layer uses softmax activation function to produce probability distribution over binary output classes - fault or fault free.

The multi-segment deep CNN is trained by binary-crossentropy loss function also called log loss. This loss function adjusts imbalanced class or labels in training data. The binary-loss-entropy loss function is defined as:

$$
\begin{aligned}
& L(\theta)=-\frac{1}{N} \sum_{i=1}^{N}\left[y_{i} \log \left(p\left(y_{i}\right)\right)+\left(1-y_{i}\right) \log (1-\right. \\
& \left.\left.p\left(y_{i}\right)\right)\right]
\end{aligned}
$$

where, $\mathrm{N}$ is the total number of data samples, $\mathrm{y}$ is a binary label of each class, $p\left(y_{i}\right)$ is predicted probability, and $\theta$ is model parameters.

The proposed deep learning model was implemented in proven open source libraries $[15][16][17][18]$. An 
appropriate architecture of deep learning network is obtained by searching an optimal combination of hyperparameters $\lambda(*)$ which minimizes the generalized error for a given learning algorithm. This could be very challenging when the dimensionality of hyperparameter search space increases. The following hyperparameters were optimized for this deep learning model before the learning process:
- Number of local convolution layers: the searchdimension of number of local convolution layers is a positive integer in the interval $[1, \ldots, 3]$. There will be at least 1 and at most 3 local convolution layer.

- Number of fully-connected convolution layers: the search-dimension of number of fully-connected

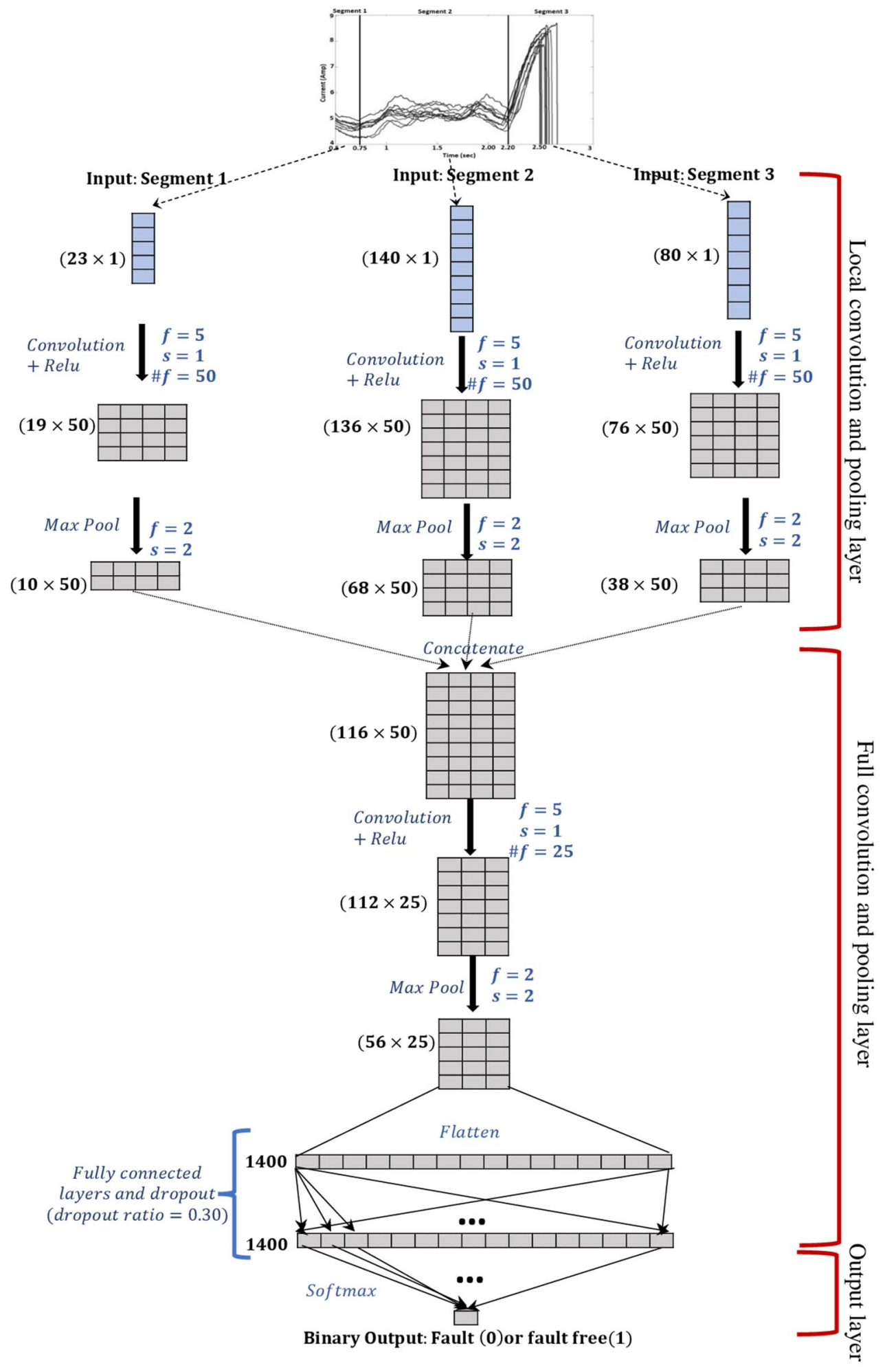

Figure 5. Archicture of Multi-segment deep CNN for railway point system 
convolution layers is a positive integer in the interval $[1, \ldots, 3]$.

- Number of flattened fully-connected/dense layers: the search-dimension of number of fully-connected dense layers is also a positive integer in the interval $[1, \ldots, 3]$.

- Size of filter in each convolution layer: search-range for filter size is a positive integer in the interval $[2, \ldots, 10]$. The temporal features are small and local, therefore a set of small filters and small stride are used to select the right hyperparameters for each layer to give greater attention to capture localized differences.

- Number of filters in each convolution layer: searchrange is a positive integer in the interval $[20, \ldots, 100]$

- Number of strides in each filter: search-range is a positive integer in the interval $[1, \ldots, 3]$

- Dropout: search-range for dropout ratio is a real number in the close interval $[0,0.70]$.

- Learning rate of the optimizer: search-range for the learning-rate is a real number in the close interval $\lceil 1 \mathrm{e}-8,1 \mathrm{e}-2\rceil$. The entire exponential range is not searched. The logarithmic transformation of this exponential range, that is $[-8, \ldots,-2]$ is searched.

Bayesian optimization was used to obtain optimal a set of hyperparameters [19]. This approach requires fewer evaluations of the learning algorithm compared to grid search and random search. It keeping track of a set of hyperparameters that has performed and haven't performed well in a certain hyperparameter space and predicts the regions of the hyperparameter space that might improve the performance of learning algorithm (minimize the error of learning algorithm). It runs new experiments in newly predicted hyperparameter space to find the next best set of hyperparameters.

\section{Classifier Performance}

The performance of the proposed deep-learning model was evaluated by cross-validation. The dataset had 1362 instances of switch rail movement by POE. The parameters in this multi-segment deep CNN model was trained and validated by these instances. Each instance is a time-series data of a switch movement which is segmented in three parts as described in Section II. The dataset was shuffled and partitioned into three parts for performance evaluation by 3 -fold cross-validation. In each fold, 2/3 of data i.e. 908 instances were utilized for training and rest $1 / 3$ data i.e. 454 instances were utilized for testing.

Ideally, a good learning algorithm must have a low bias which suggests that it fitted the data with a low error as it has efficiently captured most underlying pattern in the data. It must have low variance, which suggests that the difference in training and test error is very low or negligible which suggests consistent performance. Table 2 shows the result of 3-fold cross-validation of multi-segment deep CNN model. It can be seen that on an average the accuracy 3 -folds is 0.956 and it does not vary a lot i.e. all three folds have similar accuracy and difference in error between training and test set does not vary as well. The second fold has good performance, especially for true fault-detection rate (true-negative rate). In each fold, the test data (timeseries data of each movement) has 67 fault instances, on an average the deep-learning model detected 56 (83.11\%) faulty switch movements. Figure 6 shows the ROC curve of each fold.

\begin{tabular}{|c|c|c|c|c|}
\hline & \multicolumn{4}{|c|}{ Table 2: 3-Fold Cross-Validation } \\
\cline { 2 - 5 } Fold & Accuracy & Precision & $\begin{array}{c}\text { Recall or True } \\
\text { positive (fault- } \\
\text { free) rate }\end{array}$ & $\begin{array}{c}\text { True negative } \\
\text { (fault) rate }\end{array}$ \\
\hline 1 & 0.956 & 0.956 & 0.991 & 0.829 \\
\hline 2 & 0.961 & 0.963 & 0.993 & 0.842 \\
\hline 3 & 0.953 & 0.953 & 0.986 & 0.822 \\
\hline
\end{tabular}

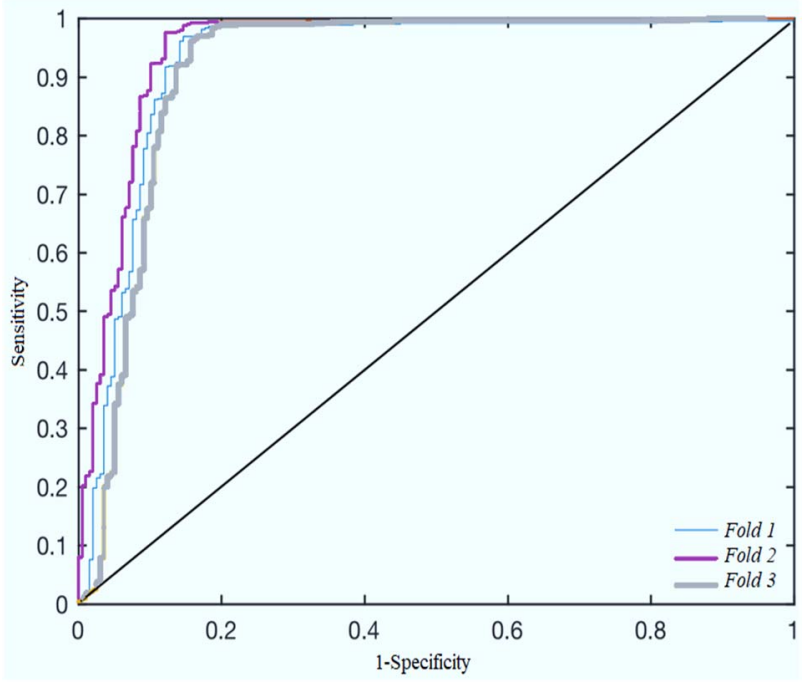

Figure 6. ROC curves for each fold

Both the hyperparameter optimization and training of multi-segment deep CNN was carried solely on CPU: Intel Core i5 $3.00 \mathrm{GHz}$ processor. In this research, this did not compare the computation power of GPUs and CPUs. The utilization of GPUs to accelerate training of $\mathrm{CNN}$ can be seen in [20][21].

\section{Conclusion}

In this paper, we introduced a deep learning algorithm based 1-dimensional convolution neural network to detect the fault in railway point operating equipment. This framework can efficiently detect the faulty switch rail movements from raw time-varying electrical-current data from current sensors. In this approach, the number of measurements for each movement (each time-series) is split into three non-overlapping segments for three different operating phases of POE. Three independent 1-D CNN is applied on each segment to capture local temporal features. The global temporal features are captured by applying 1-D $\mathrm{CNN}$ on concatenated output from the previous three local CNNs. It can be easily applied to data from multiple sensors or multiple-channels. 
The dataset containing faulty movements of a heterogeneous set of POE may affect the results if sufficient records for a particular type of POE is not available. In this paper, we have shown the fault classification result from only a sensor at POE of type HW1000/2000 series. We are confident that the valuable insight and experience gained through this research will help us to expand this approach to multiple sets of sensors on railway tracks such as pressure, inertia, and current sensors.

\section{ACKNOWLEDGMENT}

We are grateful to the Berkeley Research Group for providing valuable insight. This work is funded by the Alliance Strategic Grant of The University of Manchester.

\section{REFERENCES}

[1] W.-J. Zwanenburg, "A model for the life expectancy of railway switches and crossings for maintenance and renewal planning in asset management systems," Computers in Railways XI, 2008.

[2] M. Vileiniskis, R. Remenyte-Prescott, and D. Rama, "A fault detection method for railway point systems," Proceedings of the Institution of Mechanical Engineers, Part F: Journal of Rail and Rapid Transit, vol. 230, no. 3, pp. 852-865, 2015.

[3] F. Garciamarquez, "A reliability centered approach to remote condition monitoring. A railway points case study," Reliability Engineering \& System Safety, vol. 80, no. 1, pp. 33-40, 2003.

[4] M. A. Mchutchon, W. J. Staszewski, and F. Schmid, "Signal Processing for Remote Condition Monitoring of Railway Points," Strain, vol. 41, no. 2, pp. 71-85, 2005.

[5] F. P. G. Márquez and J. M. C. Muñoz, "A pattern recognition and data analysis method for maintenance management," International Journal of Systems Science, vol. 43, no. 6, pp. 1014-1028, 2012.

[6] N. Bolbolamiri, M. S. Sanai, and A. Mirabadi. "Time-domain stator current condition monitoring: Analyzing point failures detection by Kolmogorov-Smirnov (KS) test." Int. J. Electr. Comput. Energ. Electron. Commun. Eng 6, no. 6, pp. 587-592, 2012.

[7] T. Asada and C. Roberts, "Improving the dependability of DC point machines with a novel condition monitoring system," Proceedings of the Institution of Mechanical Engineers, Part F: Journal of Rail and Rapid Transit, vol. 227, no. 4, pp. 322-332, 2013.

[8] F. Chamroukhi, A. Same, P. Aknin, and M. Antoni, "Switch mechanism diagnosis using a pattern recognition approach," 4th IET International Conference on Railway Condition Monitoring (RCM 2008), 2008.
[9] V. Atamuradov, F. Camci, S. Baskan, and M. Sevkli, "Failure diagnostics for railway point machines using expert systems," 2009 IEEE International Symposium on Diagnostics for Electric Machines, Power Electronics and Drives, 2009.

[10] Z. Wang, W. Yan, and T. Oates, "Time series classification from scratch with deep neural networks: A strong baseline," International Joint Conference on Neural Networks (IJCNN), 2017.

[11] Y. Zheng, Q. Liu, E. Chen, Y. Ge, and J. L. Zhao, "Exploiting multichannels deep convolutional neural networks for multivariate time series classification," Frontiers of Computer Science, vol. 10, no. 1, pp. 96-112, 2015.

[12] Z. Cui, Zhicheng, W. Chen, and Y. Chen. "Multi-scale convolutional neural networks for time series classification." arXiv preprint arXiv:1603.06995 (2016).

[13] S. Yao, S. Hu, Y. Zhao, A. Zhang, and T. Abdelzaher, "DeepSense," Proceedings of the 26th International Conference on World Wide Web - WWW 17, 2017.

[14] A. Aliamiri and Y. Shen, "Deep learning based atrial fibrillation detection using wearable photoplethysmography sensor," 2018 IEEE EMBS International Conference on Biomedical \& Health Informatics (BHI), 2018.

[15] M. Abadi, A. Agarwal, P. Barham, E. Brevdo, Z. Chen, C. Citro, S. Ghemawat, "TensorFlow: Large-scale machine learning on heterogeneous systems," Software available from tensorflow. org 1, no. 2, 2015.

[16] F. Chollet. "others. 2015. Keras." 2015.

[17] F. Pedregosa, G. Varoquaux, A. Gramfort, V. Michel, B. Thirion, O. Grisel, M. Blondel et al. "Scikit-learn: Machine learning in Python." Journal of machine learning research, 12, pp. 2825-2830, 2011

[18] G. M. Kumar, and T. Head. "Scikit-optimize." Tim Head and contributors, 2017.

[19] T. Hinz, N. Navarro-Guerrero, S. Magg, and S. Wermter, "Speeding up the Hyperparameter Optimization of Deep Convolutional Neural Networks," International Journal of Computational Intelligence and Applications, vol. 17, no. 02, p. 1850008, 2018.

[20] C. Li, Y. Yang, M. Feng, S. Chakradhar, and H. Zhou, "Optimizing Memory Efficiency for Deep Convolutional Neural Networks on GPUs," SC16: International Conference for High Performance Computing, Networking, Storage and Analysis, 2016.

[21] Y. You, A. Buluç, and J. Demmel, "Scaling deep learning on GPU and knights landing clusters," Proceedings of the International Conference for High Performance Computing, Networking, Storage and Analysis on - SC 17, 2017. 